\title{
Optical Trapping of Gold Nanoparticles in Air
}

Pedersen, Liselotte Jauffred; Taheri, S. Mohammad-Reza; Schmitt, Regina; Linke, Heiner; Oddershede, Lene B.

Published in:

Nano Letters

DOI:

10.1021/acs.nanolett.5b01562

Publication date:

2015

Document version

Publisher's PDF, also known as Version of record

Citation for published version (APA):

Pedersen, L. J., Taheri, S. M-R., Schmitt, R., Linke, H., \& Oddershede, L. B. (2015). Optical Trapping of Gold Nanoparticles in Air. Nano Letters, 15(7), 4713-4719. https://doi.org/10.1021/acs.nanolett.5b01562 


\title{
Optical Trapping of Gold Nanoparticles in Air
}

\author{
Liselotte Jauffred, ${ }^{\dagger}$ S. Mohammad-Reza Taheri, ${ }^{\ddagger}$ Regina Schmitt, ${ }^{\dagger}$ Heiner Linke, ${ }^{*}{ }^{\dagger}$ \\ and Lene B. Oddershede*,§ \\ ${ }^{\dagger}$ Solid State Physics and Nanometer Structure Consortium (nmC@LU), Lund University, 22100 Lund, Sweden \\ \#Physics Department, Institute for Advanced Studies in Basic Sciences (IASBS), 45195-1159 Zanjan, Iran

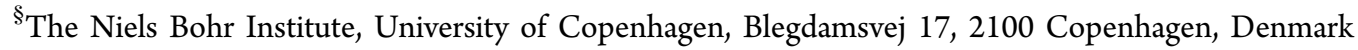

\section{Supporting Information}

ABSTRACT: Most progress on optical nanoparticle control has been in liquids, while optical control in air has proven more challenging. By utilizing an air chamber designed to have a minimum of turbulence and a single laser beam with a minimum of aberration, we trapped individual 200 to $80 \mathrm{~nm}$ gold nanoparticles in air and quantified the corresponding trapping strengths. These results pave the way for construction of metallic nanostructures in air away from surfaces.

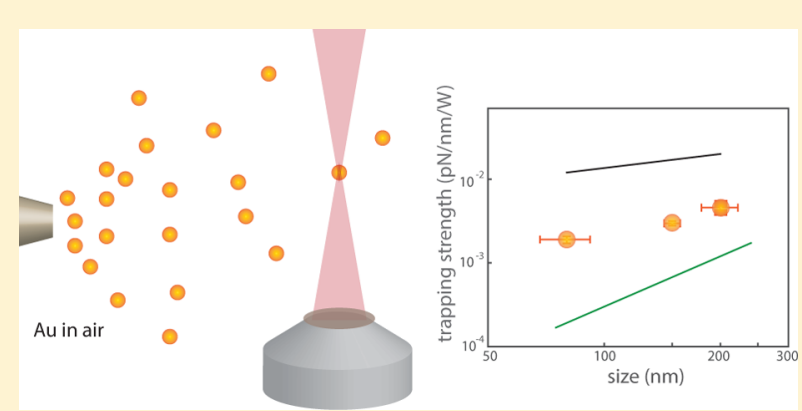

KEYWORDS: Aerosol, optical tweezers, gold nanoparticles, silica nanoparticles, power spectral analysis, laser manipulation, aerotaxy
$\mathrm{T}$ he first report on optical control of airborne particles was from Ashkin and Dziedzic, ${ }^{1}$ who, by balancing radiation pressure with gravitation, levitated airborne glycerol droplets with sizes down to $1 \mu \mathrm{m}$. Such levitation of droplets has proven highly useful for Raman spectroscopy investigations. ${ }^{2-5}$ In 1997 Omori et al. ${ }^{6}$ reported optical trapping (as opposed to levitation) of a dielectric particle in air using a single tightly focused laser beam. Since then, more progress has been accomplished on optimizing optical control of dielectric particles, ${ }^{7}$ liquid droplets have been optically trapped in air, ${ }^{8,9}$ and even multiple droplets have been trapped in air by holographic optical tweezers. ${ }^{10}$ Not just the translation but also the rotation of microparticles in air have been recently proven. ${ }^{11}$ Optical trapping of particles in air, or vacuum, was recently successfully utilized for exploring the fundamental laws of physics, for instance, the validity of the fluctuationdissipation theorem for a $150 \mathrm{~nm}$ silica particle during relaxation from a nonequilibrium state; ${ }^{12}$ also optically trapped microspheres in vacuum were cooled to the milli-Kelvin scale, ${ }^{13}$ thus allowing for the study of gravitational state reduction.

Metallic nanoparticles are highly useful as components in molecular electronics, ${ }^{14}$ and much effort has been put into gaining control over the positioning of individual metallic nanoparticles. The first optical control of individual nanoparticles in liquid was proven in $1994,{ }^{15}$ where it was shown that due to their larger inducible polarizability, gold nanoparticles trapped even more readily than polystyrene particles of similar size. Since then, the optical trapping range of goldand silver-nanoparticles has been significant expanded, ${ }^{16-18}$ and also the orientation of gold nanorods can now be optically controlled by a single laser beam. ${ }^{19,20}$ In contrast, essentially no progress has been reported on optical control of metallic nanoparticles in air or vacuum. This may be related to practical challenges, which include faster Brownian motion of aerosols because of the lower viscosity, larger optical aberration, and reduced heat dissipation in comparison to trapping in water.

The ability to manipulate and study individual metallic or semiconductor nanostructures in air or vacuum would open up many exciting opportunities, including, for example, the study of catalytic processes, of heat transfer at the solid-gas interface at the nanoscale, or of the construction of advanced nanostructures away from a surface where electron-beam lithography cannot be used. ${ }^{21,22}$ As a specific example we mention aerotaxy, the recently developed technique for growing high-quality semiconductor nanowires from $\mathrm{Au}$ seed particles in the gas phase with very high throughput. ${ }^{23}$ Because the aerotaxy technique does not use a substrate, traditional growth models cannot be directly applied. Optical trapping of individual Au particles in air could enable in situ studies of the nucleation and growth processes.

Here, we demonstrate optical trapping of individual airborne gold nanoparticles with diameters from 80 to $200 \mathrm{~nm}$ and compare to aerosol trapping of similarly sized dielectric particles. We perform a quantitative analysis of the positions visited by the trapped particles, calculate the spring constants characterizing optical trapping of metallic nanoparticles in air, and use Allan variance analysis to find the optimal measurement window. Also, we discuss challenges related to optical

Received: April 22, 2015

Revised: June 12, 2015

Published: June 17, 2015 
control of metallic nanoparticles in air, including the heating associated with nanoparticle absorption of the trapping beam.

For optical control in air we used a standard single beam optical trap based on an infrared laser beam $(\lambda=1064 \mathrm{~nm}$, Nd:YVO4, Spectra Physics BL106C) implemented in an inverted microscope (Leica, DMIRBE) as described earlier. ${ }^{24,25}$ This setup allowed us to perform simultaneous imaging and optical trapping. The laser was expanded to slightly overfill the back aperture of an oil immersion objective (NA $=1.4,100 \times$, Leica) focusing the laser into the air chamber. In order to minimize spherical aberration we used an immersion media with a refractive index of 1.57 (Cargille). ${ }^{26}$ During the experiments here presented with gold nanoparticles in air, the laser power was $\sim 700 \mathrm{~mW}$ at the exit of the laser. In water, the corresponding laser power at the sample plane was measured to be $\sim 450 \mathrm{~mW}$, but due to the extended beam waist in air, the increased internal reflection at the glass-air interface, and the aberration induced by the oil immersion objective, the power at the sample was lower in air than in water. We estimated the laser power in air in the sample plane to be $\sim 340 \mathrm{~mW}$. All results for spring constants shown were normalized with laser power at the sample plane. An air condenser placed over the chamber was used to collect the backscattered light and image it onto a quadrant photodiode (QPD, S5981, Hamamatsu) placed in the back focal plane. The acquisition frequencies were $22 \mathrm{kHz}$ for the dielectric particles and $50 \mathrm{kHz}$ for the gold nanoparticles for denser sampling at high frequencies.

To minimize turbulence we used a chamber as suggested in ref 7 consisting of three subchambers as shown in Figure 1: The

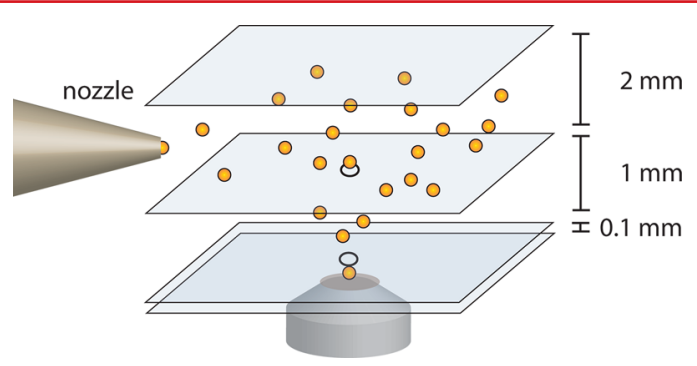

Figure 1. Sketch of the sample chamber. The sample chamber consists of three subchambers placed on top of each other and closed on all sides except where the nozzle enters. The ethanol-nanoparticle aerosol is delivered by the nebulizer through a nozzle into the upper subchamber. The upper subchamber is connected to the middle chamber by a $1 \mathrm{~mm}$ hole in the glass cover slide. The middle subchamber is again connected to the lower subchamber by a hole in the glass slide, and trapping is performed in this lower subchamber. This chamber has the advantage that turbulence is minimized in the lower trapping subchamber and only a small number of particles is delivered at the laser focus.

nanoparticle aerosol is delivered by a nebulizer in the upper chamber, a small hole $(1 \mathrm{~mm}$ in diameter drilled with a diamond drill) allows part of the airborne particles to fall in the gravitational field into the second chamber, which has less turbulence than the upper chamber. A small hole in the lower coverslip of the second chamber allows a certain fraction of these particles to fall into the third and final chamber, the trapping chamber, which is higher than the maximal working distance of the objective. The four coverslips (\#1.5) were assembled and separated by double-sided tape with different thicknesses $(100 \mu \mathrm{m}$ and $1 \mathrm{~mm})$. It is important to align the two connecting holes right on top of each other in order to have a maximal delivery of particles to the trapping chamber. Under these conditions, particles rarely entered the trap (once every $\sim 20 \mathrm{~min}$ at constant aerosol flow), enabling us to control the number of particles in the trap; as soon as a particle was registered in the trap (through a real-time QPD based analysis) we stopped the aerosol flow to minimize the risk of trapping two particles simultaneously. The concentration of nanoparticles in the aerosol was very low, corresponding to one particle for every 50-200 droplets (assuming an average droplet size of $5 \mu \mathrm{m}$, this assumption being based on visual inspection of the droplets in the microscope). We never observed stable trapping of an ethanol droplet as it evaporated instantaneously after trapping.

The aerosol consisted of a mixture of ethanol (96\%) and particle solution. For the experiments with gold nanoparticles we diluted the stock solutions of 80,150 , and $200 \mathrm{~nm}$ gold nanoparticles (all from BB International) to a final concentration of $1 \%(\mathrm{~V} / \mathrm{V})$ in ethanol. The aerosol mixture was sonicated for at least $20 \mathrm{~min}$ and vortexed several times to prevent agglomeration. We also performed experiments with PEG-coated 80 and $150 \mathrm{~nm}$ particles (from Nanopartz), the stock solutions of these were denser so they were diluted to $0.1 \%(\mathrm{~V} / \mathrm{V})$. Furthermore, we did experiments with $1 \%(\mathrm{~V} / \mathrm{V})$ silica spheres with diameters of $1010 \mu \mathrm{m}$ (Bangs Laboratories), $690 \mathrm{~nm}$, and $540 \mathrm{~nm}$ (Polysciences) and 1\% (V/V) polystyrene spheres with diameters 960, 580, and $200 \mathrm{~nm}$ (Bangs Laboratories). For all experiments we ended up with a final concentration of $0.1-1 \mathrm{nM}$ in the ethanol solution.

The ethanol-particle solution was pipetted into a nebu$\operatorname{lizer}^{7,27}$ for medical use (Omron micro air U22) and the aerosol was formed in the nozzle. Between each experiment, or after nebulizing $7 \mathrm{~mL}$ of ethanol solution, we cleaned the nebulizer filter by $20 \mathrm{~min}$ ultrasonication in Milli-Q water. We changed the sample chamber half as often. With this delivery method we observed no liquid residue on the coverglass. Such residue would cause spherical aberration that would not be consistent from one experiment to another.

We trapped gold, polystyrene, and silica nanoparticles stably in air for several minutes. The trapping depth could be varied from $0 \mu \mathrm{m}$ to more than $100 \mu \mathrm{m}$ above the surface; however, as predicted with our choice of immersion media, ${ }^{7,26}$ the trapping was measured to be optimal $\sim 12 \mu \mathrm{m}$ above the surface (unpublished data by Taheri et al.).

Using the quadrant photodiode, we measured the thermal fluctuations of the nanoparticle in the optical trap (Figure 2a). In accordance with common practice from trapping particles in liquids $^{28}$ and new results from trapping micron-particles in air, ${ }^{11}$ we assume that the positions measured in volts by the photodiode, $x_{V}$, are proportional to the metric displacement of the particle in the trap, $x_{m}: x_{m}=\beta x_{V}$.

The dynamics of a trapped particle in one translational direction $(x)$ is well described by the Langevin equation:

$$
\mathcal{F}(T, t)=\gamma \dot{x}+\kappa x+m \ddot{x}
$$

where $m$ is the mass and $\mathcal{F}(T, t)$ are the stochastic forces that depend on time, $t$, and temperature, $T$. If far from any surfaces, the friction coefficient $\gamma$ is given by Stokes law, $\gamma=3 \pi \eta d$, where $\eta$ is the viscosity of air and $d$ the diameter of the sphere. The second term, $\kappa x$, describes the force exerted on the particle by the optical trap upon an excursion, $x$, from the equilibrium position. The strength of the trap is characterized by the spring constant $\kappa$. When trapping in water, the motion is overdamped and the inertial term, $m \ddot{x}$, can safely be neglected in eq 1 ; it is 


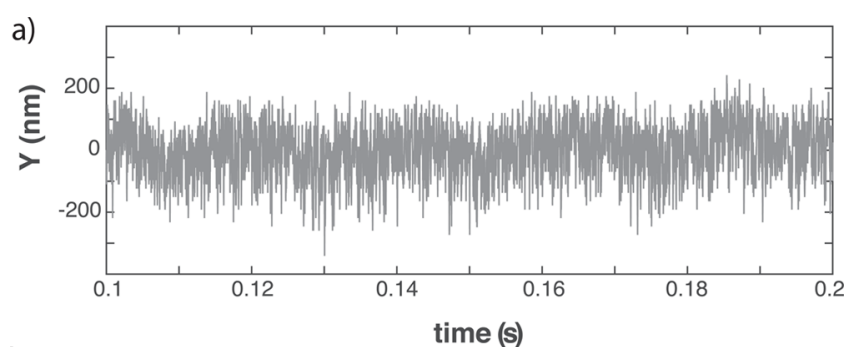

b)

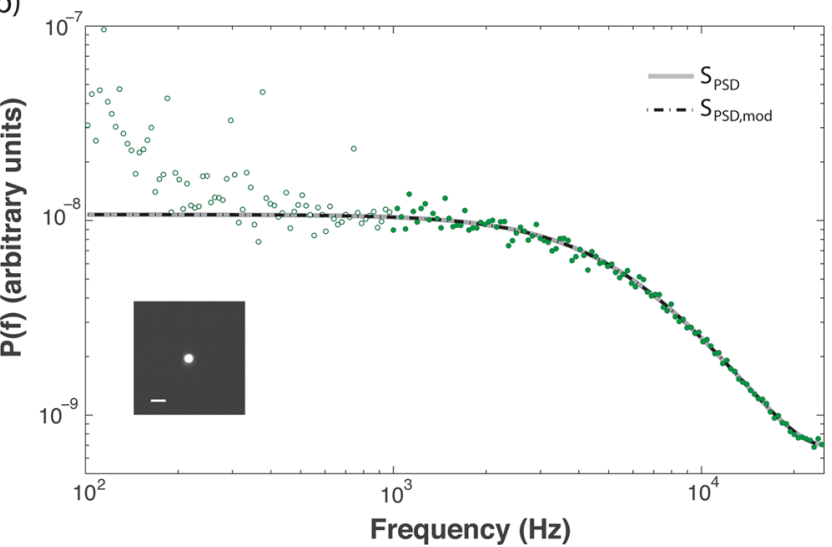

Figure 2. Analysis of the positions visited by an $80 \mathrm{~nm}$ gold nanoparticle trapped in air $12 \mu \mathrm{m}$ from the surface. (a) Position versus time in one of the lateral directions. (b) Power spectrum of the positions visited. The lines denote Lorentzian fits to data using eqs 2 (gray line) and 3 (dashed black line). The two fits appear equally good and return the corner frequencies $f_{c, E q 2}=5622 \pm 167 \mathrm{~Hz}$ and $f_{c, E q 3}=$ $5629 \pm 169 \mathrm{~Hz}$, which within the error bars are indistinguishable. The inset is a confocal image of the backscattered light, accumulated for $5 \mathrm{~s}$, from a trapped $80 \mathrm{~nm}$ gold sphere, and the scale bar is $1 \mu \mathrm{m}$.

not a priori clear whether trapping in air can also be assumed overdamped. If assuming an overdamped situation, Fourier transformation of the overdamped Langevin yields the singlesided positional power spectrum (PSD):

$$
S_{\mathrm{PSD}}(f)=\beta^{2} \frac{k_{\mathrm{B}} T}{2 \gamma \pi^{2}\left(f_{c}^{2}+f^{2}\right)}
$$

where $k_{\mathrm{B}}$ is the Boltzmann factor and the corner frequency, $f_{c}$, is the ratio between the trap stiffness, $\kappa$, and the friction coefficient, $\gamma: f_{c}=\kappa /(2 \pi \gamma)$. A typical powerspectrum from a trapped gold nanoparticle in air is shown in Figure $2 b$, and as apparent from this figure, a simple Lorentzian function (eq 2) fits quite well to data from a metallic nanoparticle trapped in air. The power spectral analysis directly returns $f_{c}$ and hence $\kappa$, as well as the conversion factor $\beta$.

The calibration method outlined above assumes that the dynamics of the trapped particle is overdamped, which is not necessarily the case in air, although we do estimate the Reynolds number to be rather low $\left(10^{-7}\right)$. To better describe the motion of a metallic nanoparticle trapped in air it may be more correct to include the inertial term in the Langevin equation (eq 1). This causes the power spectrum to be modified to the following expression: ${ }^{2}$

$$
S_{\mathrm{PSD}, \bmod }(f)=\beta^{2} \frac{2 k_{\mathrm{B}} T}{\kappa} \frac{\Omega^{2} \gamma^{\prime}}{\left(4 \pi^{2} f^{2}-\Omega^{2}\right)^{2}+4 \pi^{2} \gamma^{\prime 2} f^{2}}
$$

where $\Omega=(\kappa / m)^{1 / 2}$ and $\gamma^{\prime}=\gamma / m C$. $C$ is the Cunningham slip correction factor to the drag coefficient; it is necessary to invoke this if the mean free path becomes larger than the size of the particle. For a $200 \mathrm{~nm}$ gold nanoparticle's trajectory we find that the quartic term of $f$ in eq 3 is negligible when $f \ll \Omega \approx$ $150 \mathrm{kHz}$, which is well above the corner frequencies found here (which are a couple of $\mathrm{kHz}$ ). Hence, eq 3 collapses to eq 2 in the relevant trapping regime, and the simpler eq 2 can safely be used to fit the data. This is also confirmed by Figure $2 b$, which shows that the two fits by eqs 2 and 3 coincide and return similar values of the corner frequency. The same conclusion can be reached by calculating the characteristic time, $\tau$, for loss of kinetic energy through friction. ${ }^{29}$ For the gold nanoparticles, $\tau$ $=m / \gamma \approx 2 \mu \mathrm{s}$, which is well below our sampling time of $20 \mu \mathrm{s}$. Likewise for the $1 \mu \mathrm{m}$ silica beads we get $\tau=m / \gamma \approx 3 \mu \mathrm{s} \ll 45$

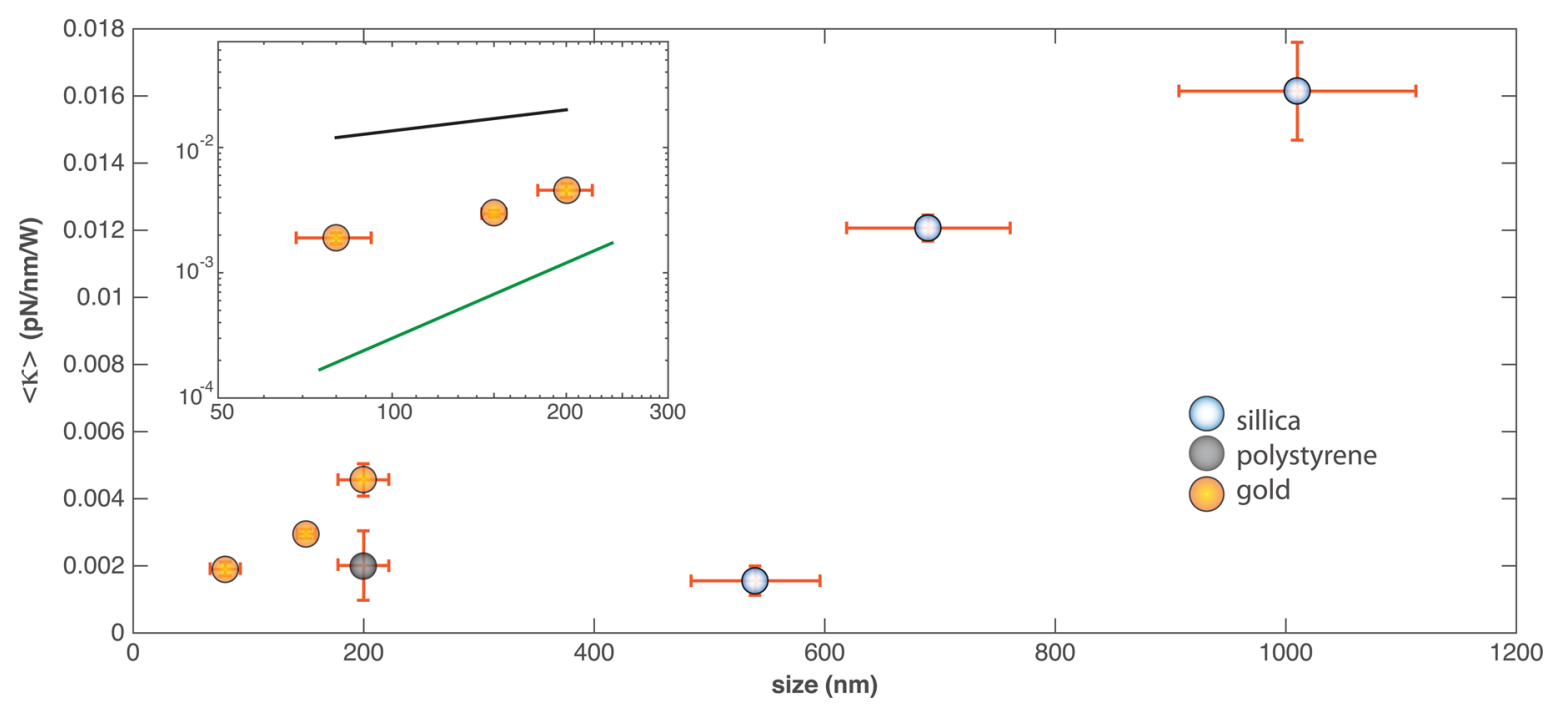

Figure 3. Trapping strength $\langle\kappa\rangle$ (normalized with laser power) versus particle size for gold nanoparticles (yellow), silica particles (blue), and polystyrene particles (gray). Each value of $\langle\kappa\rangle$ shown is the mean value from at least 5 independent experiments, and the error bars show one standard deviation. The error bars on particle diameter are as given by the manufacturer. The inset shows a double logarithmic plot of $\langle\kappa\rangle$ versus diameter for the gold nanoparticles. The green line has a slope of 2 , and the black line shows the scaling of $\kappa$ for gold nanoparticles of similar size in water. $^{16}$ 
$\mu \mathrm{s}$ ( $45 \mu \mathrm{s}$ is the sampling time for the silica beads). Based on these arguments, we analyzed our data with the programs from ref 30 (shown by the full gray line in Figure $2 b$ ).

The trapping strength, $\kappa$, as a function of particle diameter is shown in Figure 3 for different particle types. As expected, the spring constant characterizing optical trapping of individual gold nanoparticles in air increases with particle size. For similarly sized particles, gold nanoparticles trap more strongly than polystyrene nanoparticles and also stronger than much larger $(450 \mathrm{~nm})$ silica nanoparticles.

As the concentration of nanoparticles was very low (on average 1 particle per $\sim 100$ droplets) and as the error bars on $\kappa$ are rather small, it is very likely that most often we trap only a single particle at a time. We also did experiments with PEGcoated particles; the purpose of these experiments was to prevent the particles from aggregating while in the liquid phase because PEG coating is known to prevent aggregation in solution. From the bright field images it appears the particles were delivered to the trap in a liquid droplet. Thus, the PEG coating might prevent agglomeration until the droplet is trapped, at which point the ethanol (and possibly the PEG too) evaporates immediately. The time scale of evaporation (microseconds) is several orders of magnitude shorter than the observation time scale of the experiment (tens of seconds); hence, all reported results are from a situation where ethanol has evaporated. As shown in Figure S1, the results from aerosol trapping of PEG coated gold nanoparticles were indistinguishable from trapping noncoated gold nanoparticles, and the data in Figure 3 is from both types of experiments.

Furthermore, the trapping characteristics of the two lateral directions are similar (see Figure S2). If the metallic nanoparticles had been elongated (for example, because a small number of particles stuck together), and not spherical, they would have aligned along the laser polarization (which is one of the lateral directions measured), and a difference in the two orthogonal lateral directions would have been observed. ${ }^{19}$ As the spring constants, characterizing the two lateral directions, within the error bars are identical, it is quite likely that the investigated metallic nanoparticles were spherical as expected. A single trapped $80 \mathrm{~nm}$ gold nanoparticle was visible in reflection confocal microscopy but not in bright field microscopy, and the $200 \mathrm{~nm}$ gold nanoparticles were also visible in bright field.

It was easier to stably trap airborne polystyrene particles, also at relatively low laser powers, than trapping airborne gold nanoparticles, and we performed a systematic study of how the spring constant, $\kappa$, characterizing aerosol polystyrene particle trapping in the lateral directions varied with laser power. The laser power at the sample plane was varied from 100 to 260 $\mathrm{mW}$, and $\kappa$ versus laser power is shown in Figure S3. Interestingly, in the investigated power interval $\kappa$ decreases linearly with increasing laser power, which is opposite of the behavior of $\kappa$ versus power observed for optical trapping of particles, both polystyrene and metallic, in water. Our results from quantifying $\kappa$ versus laser power for polystyrene particles in air are consistent with theoretical results for aerosol trapping, ${ }^{31}$ which predict that in a certain power regime the axial strength characterizing aerosol trapping should decrease with laser power.

The gold nanoparticles investigated here are in the Rayleigh regime where $d \ll \lambda, \lambda$ being the wavelength of the trapping laser $(1064 \mathrm{~nm})$. In this regime, a gold nanoparticle can be considered a dipole in the electric field, and the lateral gradient force, $F_{\text {grad }}$ is proportional to the polarizability, $\alpha: F_{\text {grad }} \propto \alpha .^{15}$ The polarizability is proportional to the polarizable volume, $V$, and is given by the Clausius-Mossotti relationship:

$$
\alpha=V \frac{1-\epsilon_{r}}{1+2 \epsilon_{r}}
$$

where $\epsilon_{r}$ is the ratio between the permittivity of air and gold at the relevant wavelength. For small particles, one can assume that the entire particle is polarized. However, for particles with radii larger than the skin depth of gold, $\delta=23 \mathrm{~nm}^{15}$ at 1064 $\mathrm{nm}$, the field intensity decays exponentially inside the particle, and the polarized volume is instead $V^{\prime}:^{15}$

$$
V^{\prime}=4 \pi \int_{0}^{a} r^{2} \exp \left(\frac{r-a}{\delta}\right) \mathrm{d} x
$$

where $a$ is the radius of the particle. This correction results in the scaling: $F_{\text {grad }} \propto a^{2}$, which intuitively corresponds to the fact that only an outer shell of the particle is polarized. For this reason, one would expect $\kappa$, from particles with radii larger than the skin depth, to scale as $a^{2}$. However, the plot of $\langle\kappa\rangle$ versus diameter on double logarithmic axes in the inset of Figure 3 clearly shows that the scaling is smaller than quadratic (the green line has a slope of 2). Rather, it seems the scaling is quite similar to the scaling observed from trapping gold nanoparticles in water ${ }^{16}$ (shown by a black line in the inset). The fact that $\langle\kappa\rangle$ does not scale as $a^{2}$ is probably due to significant spherical aberration at the focus because it was shown that if great care is taken in water to remove spherical aberration, then a scaling exponent of 2 can be obtained while trapping gold nanoparticles in water. ${ }^{18}$ Of interest is also the fact that the $\langle\kappa\rangle$ s' here found characterizing optical trapping of gold nanoparticles are approximately $1 / 10$ (corrected for laser power) of the trapping strengths obtained for gold nanoparticles ${ }^{16,18}$ or other spherical nanoparticles ${ }^{17,32,33}$ in water. Figure S4 visualizes this stronger trapping in water than in air by showing simulated trajectories of an $80 \mathrm{~nm}$ gold nanoparticle traveling in air and in water, respectively.

For dielectric spheres with diameters from $200 \mathrm{~nm}$ to $1 \mu \mathrm{m}$ $\langle\kappa\rangle$ is expected to increase with $d$ until it reaches the lateral extent of the focus $(\sim 1 \mu \mathrm{m}) .{ }^{34}$ This is also observed in Figure 3 for the silica particles. In accordance with the early results of trapping gold versus dielectric nanoparticles in liquid ${ }^{15}$ we find that also in air, gold nanoparticles have larger $\kappa$ than similarly sized dielectric particles.

All parts of the equipment are prone to drift and noise during optical trapping of the airborne nanoparticles and lowfrequency drift is quite visible in the long time series. Allan variance analysis is a very useful tool for quantifying noise in optical trapping, and it pinpoints the optimal data acquisition time where the benefit of acquiring more data points is exactly counteracted by drift in the system. ${ }^{35}$ Figure $4 \mathrm{a}$ shows Allan variance calculated as a function of measurement time for both an $80 \mathrm{~nm}$ gold nanoparticle and a $1 \mu \mathrm{m}$ silica particle. The global minimum in each of the curves denote the optimal measurement times, which turn out to be $0.04 \mathrm{~s}$ and $0.2 \mathrm{~s}$ for the gold and silica particles, respectively. In accordance with this, the corresponding scatterplots of time series with the optimal length found by Allan variance analysis (Figure 4b,c) appear quite spherical and without drift (and so do the time series, data not shown). This analysis also carries the information that optical trapping of nanoparticles in air is more prone to drift and instrument noise than optical trapping 

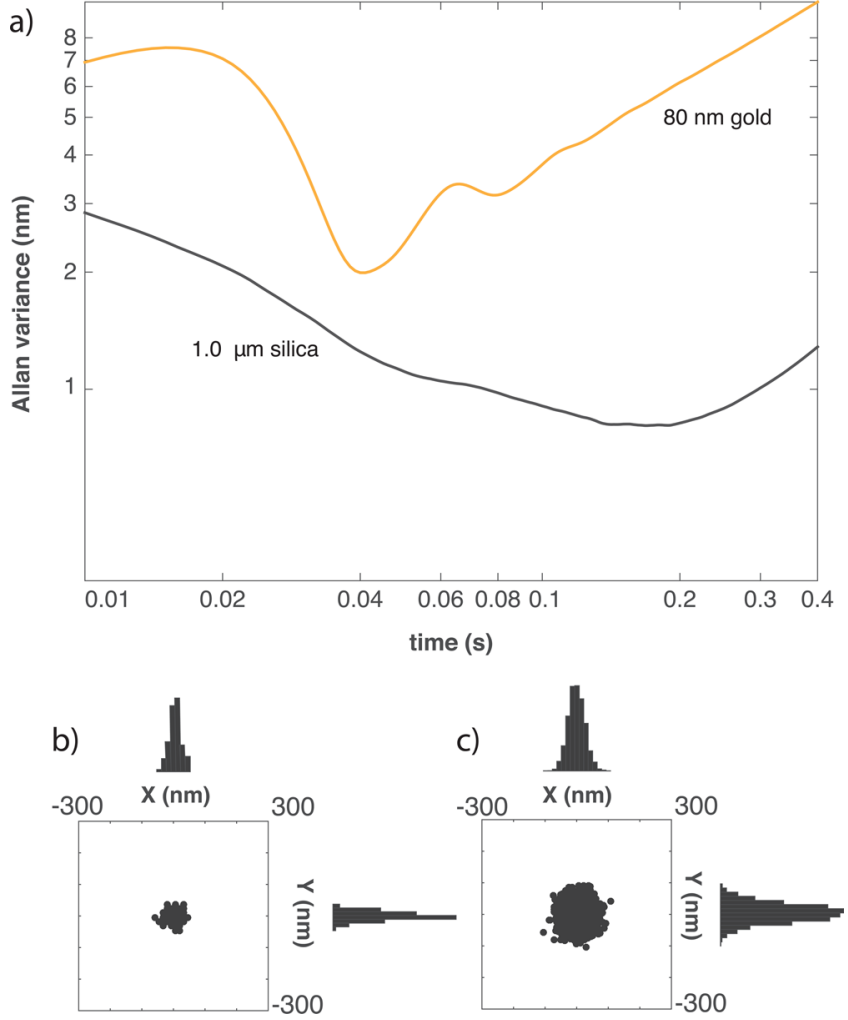

Figure 4. Allan variance analysis of time series from optical trapping of airborne nanoparticles. (a) Allan variance as a function of measurement time for a $80 \mathrm{~nm}$ gold nanoparticle and for a $1 \mu \mathrm{m}$ silica particle. The global minimum in the Allan variance curves pinpoint the optimal measurement time where the benefit of acquiring additional data points is counteracted by mechanical drift and noise in the system. For $80 \mathrm{~nm}$ gold the optimal measurement time is $0.04 \mathrm{~s}$, for $1 \mu \mathrm{m}$ silica 0.2 s. $(b, c)$ Scatterplots and corresponding histograms from time series of optical trapping of airborne $80 \mathrm{~nm}$ gold (b) and $1 \mu \mathrm{m}$ silica (c). The lengths of the time series shown are exactly the optimal times found by Allan variance analysis (as shown in a).

of nanoparticles in liquid because the optimal measurement times for nanoparticles trapped with similar laser powers in water were found to be $2-4 \mathrm{~s}$ for micron-sized dielectric particles and $\sim 0.5 \mathrm{~s}$ for metallic nanoparticles, ${ }^{36}$ thus significantly longer than in air.

Due to their plasmonic properties, irradiated metallic nanoparticles absorb part of the incident radiation and liberate the absorbed energy as heat or radiation to their surroundings. However, for the temperatures of interest here, heat dissipation by radiation is negligible. The exact temperature rise depends on particle size, material, shape, orientation, incident laser power, wavelength, and thermal conductivity of the medium. ${ }^{37-40}$ To our knowledge, all quantitative experimental measurements of the temperature profile around irradiated nanoparticles were performed in water. Inspired by refs 37 and 38 we used Mie theory ${ }^{41}$ to calculate the absorption and scattering cross sections for a $200 \mathrm{~nm}$ gold nanoparticle in water and in air as a function of irradiating wavelength. The results are shown in Figure S5, the equations used are given in the supportive text. Figure 5A shows the absorption cross section (blue line), the scattering cross section (red line), and their sum (black dashed line), the extinction cross section, as a function of particle size for a gold nanoparticle in air irradiated by $1064 \mathrm{~nm}$. The extinction cross section in water is also shown a)

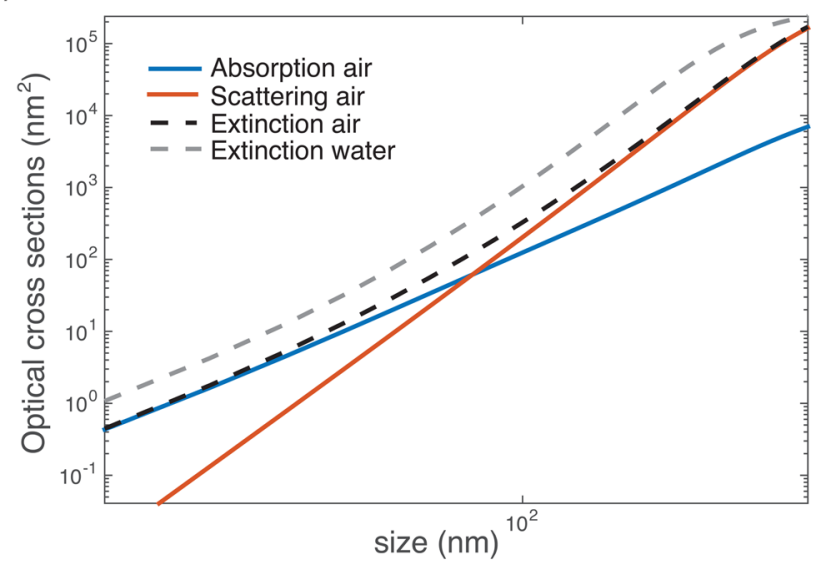

b)

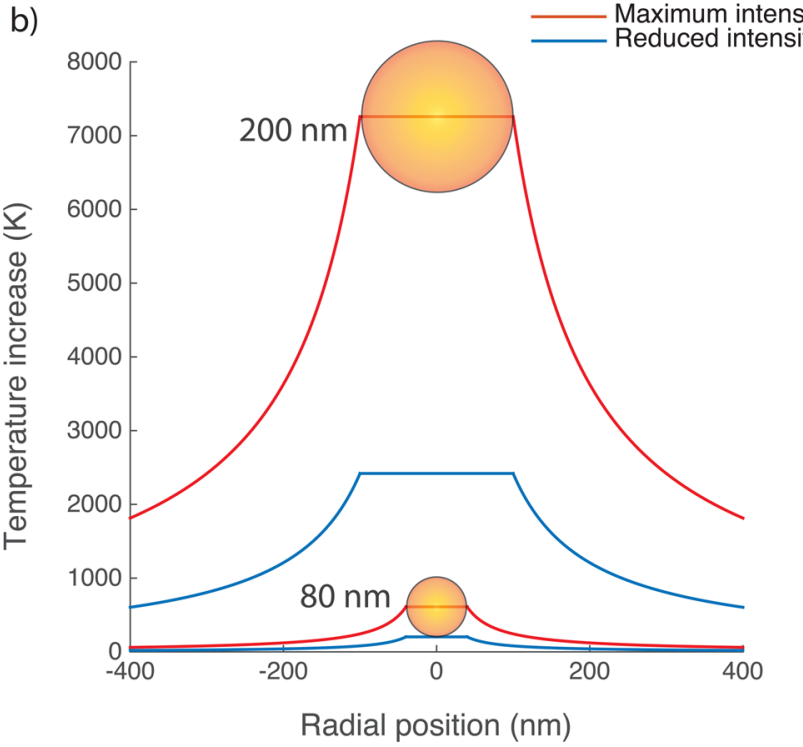

Figure 5. Theoretical estimation of the absorption and associated temperature rise of gold nanoparticles trapped in air by a $1064 \mathrm{~nm}$ laser delivering $340 \mathrm{~mW}$ at the sample plane. (a) Absorption (blue line), scattering (red line), and extinction (black dashed line) cross sections as a function of particle diameter calculated by Mie theory ${ }^{41}$ as shown in Supporting Information. For comparison the extinction cross section for gold in water is also shown (gray dashed line). (b) Calculated temperature profiles of an $80 \mathrm{~nm}$ and a $200 \mathrm{~nm}$ gold nanoparticle at positions with the highest possible intensity (red lines) and in positions with one-third of the maximum intensity (blue lines).

(gray dashed line). As apparent from these figure, the absorption, scattering, and extinction cross sections of a gold nanoparticle irradiated by $1064 \mathrm{~nm}$ are larger in water than in air. Nevertheless, due to the significantly lower thermal conductance of air $(0.024 \mathrm{~W} / \mathrm{mK})$ than of water $(0.6 \mathrm{~W} /$ $\mathrm{mK})$, the temperature rise around an airborne trapped metallic nanoparticle is significantly higher than around a similar particle trapped in water.

Assuming we know the absorption cross sections and the thermal conductivity of the media, we calculated the heating of airborne gold nanoparticles with diameters of 80, 150, and 200 $\mathrm{nm}$ to be $1.8,9.6$, and $21 \mathrm{~K} / \mathrm{mW}$, respectively (see Supporting Information for details). The corresponding values in water are $0.15,0.88$, and $2.0 \mathrm{~K} / \mathrm{mW}$, respectively. Hence, the heating of airborne particles is expected to be more than 10 times larger than in water. If the laser intensity at the location is known, the 
temperature increase at the surface of the metallic particle can be estimated. This is done in Figure $5 b$, which shows the temperature profiles around irradiated airborne gold nanoparticles with diameters of 80 and $200 \mathrm{~nm}$ assuming that the particle is located at the peak of a beam with a perfect Gaussian intensity profile. If a $200 \mathrm{~nm}$ particle were located at such a peak of an unaberrated perfect intensity distribution (red curve in Figure $5 \mathrm{~b}$ ), then the temperature elevation at its surface would be $\sim 7000 \mathrm{~K}$. This, however, is not a realistic scenario because the laser focus is highly aberrated; the aberration is caused by the fact that the objective is optimized for focusing visible light in water, not for focusing $1064 \mathrm{~nm}$ light in air. Such aberration is also theoretically predicted ${ }^{31}$ in air and will cause the overall intensity to be significantly weaker than if the laser beam had been focused in water by a similar objective. However, even in water, the focus of an infrared laser beam has been found to be severely aberrated and gold nanoparticles trapped in water in such an aberrated focus have been observed to remain stably trapped in lobes with relatively low intensity, in particular the larger particles were prone to off-maximum trapping. ${ }^{25}$ This is further supported by a study of semiconducting nanoparticles (quantum dots), which only rarely $\left(<5 \%\right.$ of the time) are trapped in the highest intensity region. ${ }^{42}$ If one assumes that the intensity at the position of an aerosol trapped gold nanoparticle is $1 / 3$ of the maximum intensity, then the temperature profiles for 80 and $200 \mathrm{~nm}$ particles would be as shown by the blue curves in Figure $5 \mathrm{~b}$. The boiling point of gold is $3243 \mathrm{~K}$, and we never observed boiling or fragmenting of a trapped $200 \mathrm{~nm}$ gold nanoparticle; hence, it is realistic that the high aberration of the focus causes the trapped large metallic nanoparticles to be located at positions where the intensity is significantly lower than at the peak of a perfect Gaussian intensity profile.

The standard deviation characterizing the Gaussian distributed positions visited by the trapped nanoparticle in the harmonic trap gives a fingerprint about the magnitude of typical excursions. Assuming that the axial trap stiffness is a factor of 5 weaker than the lateral stiffness, ${ }^{34}$ a typical standard deviation for a gold nanoparticle in the axial direction will be on the order of $\sim 100 \mathrm{~nm}$. Typical excursions of a gold nanoparticle in all three translational directions, both in water and in air, are shown in Figure S4. The intensity profile of an unaberrated laser beam falls off at distances much longer than the typical Brownian fluctuations (as shown in Figure S6). Hence, the Brownian excursions in the trap cannot explain that the temperature increase in the experiment appears much smaller than theoretically estimated. As argued above, the real explanation most likely is that the stable trapping position is not in the center of an unaberrated focus but rather in a lower intensity lobe. ${ }^{25,43}$

All the spherical gold nanoparticle sizes investigated here, with diameters from 80 to $200 \mathrm{~nm}$, could stably individually be optically trapped in air. It is likely that also aerosol particles beyond this size interval and with different composition and shapes can be stably trapped, and future efforts will explore the limits. In comparison to similarly sized silica or polystyrene particles, gold nanoparticles trap more strongly in air, however, with spring constants that are approximately ten times smaller than for trapping similar particles in water. When trapping in air, there appears to be a larger drift than in water, possibly due to the more aberrated laser beam, and Allan variance analysis does indeed show that the optimal data acquisition time for particles in air is shorter than in water. Airborne trapped metallic nanoparticles heat significantly more than similar particles trapped in water, and it will be quite interesting to further explore the question of exactly how much an irradiated metallic nanoparticle heats in air, and to compare its hot Brownian motion ${ }^{44}$ to that of a similar particle in water. For this to succeed, however, it is necessary to map out the intensity distribution of the aberrated laser beam in air and to know exactly at which location a stably trapped particle is located. Hopefully, future efforts will address these topics and utilize the presented techniques to construct, investigate, and control metallic nanostructures in air, away from the surfaces.

\section{ASSOCIATED CONTENT}

\section{S Supporting Information}

Figure S1 showing the effect of PEG coating the gold nanoparticles, Figure S2 providing trapping characteristics in each of the two lateral directions, Figure S3 showing the correlation between trap stiffness and laser power for a polystyrene microparticle trapped in air, Figure S4 showing simulations of the positions visited by a trapped $200 \mathrm{~nm}$ gold nanoparticle in air, Figure S5 providing the absorption and scattering cross sections for a $80 \mathrm{~nm}$ gold nanoparticle in air and water, respectively, Figure S6 showing the axial intensity decay of an unaberrated laser beam as well as the intensity felt by a particle performing thermal fluctuations in the trap, and supporting text detailing the calculations of irradiated gold nanoparticle temperature elevation. The Supporting Information is available free of charge on the ACS Publications website at DOI: $10.1021 /$ acs.nanolett.5b01562.

\section{AUTHOR INFORMATION}

\section{Corresponding Authors}

*E-mail: heiner.linke@ftf.lth.se.

*E-mail: oddershede@nbi.dk.

\section{Notes}

The authors declare no competing financial interest.

\section{ACKNOWLEDGMENTS}

The author acknowledge fruitful discussions and advice from J. Johansson, S. N. S. Reihani, and P. M. Bendix and financial support from the Excellence Program at the University of Copenhagen, from the Knut and Alice Wallenberg Foundation and the Nanometer Structure Consortium, Lund University (nmC@LU).

\section{REFERENCES}

(1) Ashkin, A.; Dziedzic, J. M. Science 1975, 187, 1073-1075.

(2) Thurn, R; Kiefer, W. Appl. Spectrosc. 1984, 78-83.

(3) Biswas, A.; Latifi, H. Phys. Rev. A 1989, 40, 7413-7416.

(4) Lettieri, T. R.; Jenkins, W. D.; Swyt, D. a. Appl. Opt. 1981, 20, 2799-805.

(5) Lettieri, T. R; Preston, R. E. Opt. Commun. 1985, 54, 349-352.

(6) Omori, R.; Kobayashi, T.; Suzuki, A. Opt. Lett. 1997, 22, 816818

(7) Taheri, S. M. R.; Sadeghi, M.; Madadi, E.; Reihani, S. N. S. Opt. Commun. 2014, 329, 196-199.

(8) Magome, N.; Kohira, M. I.; Hayata, E.; Mukai, S.; Yoshikawa, K. J. Phys. Chem. B 2003, 107, 3988-3990.

(9) Hopkins, R. J.; Mitchem, L.; Ward, A. D.; Reid, J. P. Phys. Chem. Chem. Phys. 2004, 6, 4924-4927.

(10) Burnham, D. R.; McGloin, D. Opt. Express 2006, 14, 41754181.

(11) Arita, Y.; Mazilu, M.; Dholakia, K. Nat. Commun. 2013, 4, 2374. 
(12) Gieseler, J.; Quidant, R.; Dellago, C.; Novotny, L. Nat. Nanotechnol. 2014, 9, 358-364.

(13) Li, T.; Kheifets, S.; Raizen, M. G. Nat. Phys. 2011, 7, 527-230.

(14) Osorio, H. M.; Cea, P.; Ballesteros, L. M.; Gascón, I.; MarquesGonzalez, S.; Nichols, R. J.; Pérez-Murano, F.; Low, P.; Martin, S. J. Mater. Chem. C 2014, 2, 7348-7355.

(15) Svoboda, K.; Block, S. M. Opt. Lett. 1994, 19, 930-932.

(16) Hansen, P. M.; Bhatia, V. K.; Harrit, N.; Oddershede, L. B. Nano Lett. 2005, 5, 1937-1942.

(17) Bosanac, L.; Aabo, T.; Bendix, P. M.; Oddershede, L. B. Nano Lett. 2008, 8, 1486-1491.

(18) Hajizadeh, F.; Reihani, S. N. S. Opt. Express 2010, 18, 551-559.

(19) Selhuber-Unkel, C.; Schubert, I. Z. O.; Sönnichsen, C.; Oddershede, L. B. Nano Lett. 2008, 8, 2998-3003.

(20) Ruijgrok, P.; Verhart, N.; Zijlstra, P.; Tchebotareva, a.; Orrit, M. Phys. Rev. Lett. 2011, 107, 1-4.

(21) Yue, W.; Wang, Z.; Yang, Y.; Chen, L.; Syed, A.; Wong, K.; Wang, X. J. Micromech. Microeng. 2012, 22, 125007.

(22) Mendes, P. M.; Jacke, S.; Critchley, K.; Plaza, J.; Chen, Y.; Nikitin, K.; Palmer, R. E.; Preece, J. a.; Evans, S. D.; Fitzmaurice, D. Langmuir 2004, 20, 3766-3768.

(23) Heurlin, M.; Magnusson, M. H.; Lindgren, D.; Ek, M.; Wallenberg, L. R.; Deppert, K.; Samuelson, L. Nature 2012, 492, 90-94.

(24) Richardson, A.; Reihani, S. N. S.; Oddershede, L. B. Proc. SPIE 2006, 6326, 632628.

(25) Kyrsting, A.; Bendix, P. M.; Oddershede, L. B. Nano Lett. 2013, 13, 31-5.

(26) Reihani, S. N. S.; Oddershede, L. B. Opt. Lett. 2007, 32, 19982000.

(27) Summers, M. D.; Burnham, D. R.; McGloin, D. Opt. Express 2008, 16, 7739-7747.

(28) Ott, D.; Reihani, S. N. S.; Oddershede, L. B. Rev. Sci. Instrum. 2014, 85, 053108 .

(29) Berg-Sørensen, K.; Flyvbjerg, H. Rev. Sci. Instrum. 2004, 75, $594-612$.

(30) Hansen, P. M.; Tolić-Nørrelykke, I. M.; Flyvbjerg, H.; BergSørensen, K. Comput. Phys. Commun. 2006, 174, 518-520.

(31) Burnham, D. R.; McGloin, D. J. Opt. Soc. Am. B 2010, 28, $2856-2864$.

(32) Jauffred, L.; Oddershede, L. B. Nano Lett. 2010, 10, 1927-1930.

(33) Jauffred, L.; Richardson, A. C.; Oddershede, L. B. Nano Lett. 2008, 8, 3376-3380.

(34) Rohrbach, A. Phys. Rev. Lett. 2005, 95, 168102.

(35) Czerwinski, F.; Richardson, A. C.; Oddershede, L. B. Opt. Express 2009, 17, 13255-13269.

(36) Jauffred, L.; Sletmoen, M.; Czerwinski, F.; Oddershede, L. B. Proc. SPIE 2010, 7762, 776226.

(37) Seol, Y.; Carpenter, A. E.; Perkins, T. T. Opt. Lett. 2006, 31, 2429-2431.

(38) Bendix, P. M.; Reihani, S. N. S.; Oddershede, L. B. ACS Nano 2010, 4, 2256-2262.

(39) Ma, H.; Bendix, P. M.; Oddershede, L. B. Nano Lett. 2012, 12, 3954-60.

(40) Ma, H.; Tian, P.; Pello, J.; Bendix, P. M.; Oddershede, L. B. Nano Lett. 2014, 14, 612-619.

(41) Mie, G. Ann. Phys. 1908, 3, 377-445.

(42) Jauffred, L.; Kyrsting, A.; Arnspang, E. C.; Reihani, S. N. S.; Oddershede, L. B. Nanoscale 2014, 6, 6997-7003.

(43) Kyrsting, A.; Bendix, P. M.; Stamou, D. G.; Oddershede, L. B. Nano Lett. 2011, 3, 888-892.

(44) Rings, D.; Schachoff, R.; Selmke, M.; Cichos, F.; Kroy, K. Phys. Rev. Lett. 2010, 105, 8-11. 